\title{
Design Method for Single-Blade Centrifugal Pump Impeller*
}

\author{
Yasuyuki NISHI $^{* *}$, Ryota FUJIWARA ${ }^{* * *}$ and Junichiro FUKUTOMI ${ }^{* * *}$ \\ **ShinMaywa Industries, Ltd., \\ 14 Takumidai, Ono-shi, Hyogo, 675-1322 Japan \\ E-mail: nishi.y@sa.shinmaywa.co.jp \\ ***Department of Mechanical Engineering, The University of Tokushima, \\ 2-1 Minamijosanjima, Tokushima, 770-8506 Japan
}

\begin{abstract}
The sewage pumps are demanded a high pump efficiency and a performance in passing foreign bodies. Therefore, the impeller used by these usages requires the large passed particle size (minimum particle size in the pump). However, because conventional design method of pump impeller results in small impeller exit width, it is difficult to be applied to the design of single-blade centrifugal pump impeller which is used as a sewage pump. This paper proposes a design method for single-blade centrifugal pump impeller. As a result, the head curve of the impeller designed by the proposed design method satisfied design specifications, and pump efficiency was over $62 \%$ more than conventional single-blade centrifugal pump impeller. By comparing design values with CFD analysis values, the suction velocity ratio of the design parameter agreed well with each other, but the relative velocity ratio did not agree due to the influence of the backflow of the impeller entrance.
\end{abstract}

Key words: Turbomachinery, Centrifugal Impeller, Single Blade, Design Method, CFD

\section{Introduction}

Concurrently with progress in computational fluid dynamics technology, the CFD analysis is used widely in hydraulic design of a pump impeller ${ }^{(1)}$. In hydraulic design of a pump impeller, however, it is a common procedure to perform schematic design by some method prior to detailed CFD analysis and then perform detail design by modifying details using CFD analysis. For this reason, a large portion of impeller design is established before the application of CFD analysis and hence the portion the schematic design occupies in impeller design is considerably large. In a conventional schematic design, it is a common practice to integrate into design diagrams pieces of design data of impellers that exhibits satisfactory results in terms of pump efficiency and select design constants corresponding to the specific speed of a design point to design an impeller. One of the typical methods is to determine major dimensions of an impeller on the basis of Stepanoff's design diagrams ${ }^{(2)}$. However, Stepanoff's method refers to a case of a blade outlet angle of $22.5^{\circ}$. Therefore, a difference of the pump efficiency and the effect of the number of blades are not clear.

On the other hand, satisfactory performance in passing foreign bodies is required of sewage pumps handling fluids containing solids and fibrous foreign bodies. For this reason, it is critical in evaluating pump performance to secure as large a passed particle size (the minimum particle size for the flow channel in the pump) as possible with reference to the

\footnotetext{
*Received 17 Sep., 2009 (No. T2-09-0032) Japanese Original : Trans. Jpn. Soc. Mech. Eng., Vol.75, No.754, B (2009), pp.1320-1328 (Received 9 Jan., 2009) [DOI: 10.1299/jfst.4.786]
} 
pump discharge diameter. However, designing the impeller by conventional design methods ${ }^{(2)(3)}$ results in an impeller having a blade outlet width that is extremely small compared to the pump discharge diameter; therefore, it cannot be applied to a single-blade centrifugal impeller used as a sewage pump. Reducing the number of blades to increase the passed particle size results in the backflow at the blade inlet section and increased loss in the flow channel, which in turn makes a sewage pump less efficient than a multi-blade pump. To solve this problem, the authors proposed a new sewage impeller that secures a large passed particle size with a single blade, showing that its performance characteristics is better than that of a conventional type. In addition, the authors showed the behavior of radial thrust experimentally that poses a design problem, and they clarified the relationship between internal flow and loss ${ }^{(4)-(6)}$.

However, the difference in performance of newly designed impeller from that of a conventional single-blade impeller has not yet been clear and a general impeller design method has not been established under a condition in which the passed particle size is specified in this manner.

Under these circumstances, this study aims at establishing a method of designing a single-blade centrifugal impeller; while the study takes fluctuating radial thrust into consideration, it specifies the passed particle size, that is, the blade width, and proposes a theoretical method of performing schematic design of an impeller in the main. The authors examined the performance and radial thrust of a single-blade centrifugal impeller designed on the basis of the proposed design method from the viewpoint of experiments and CFD analysis, the results of which are presented below.

\section{Nomenclature}

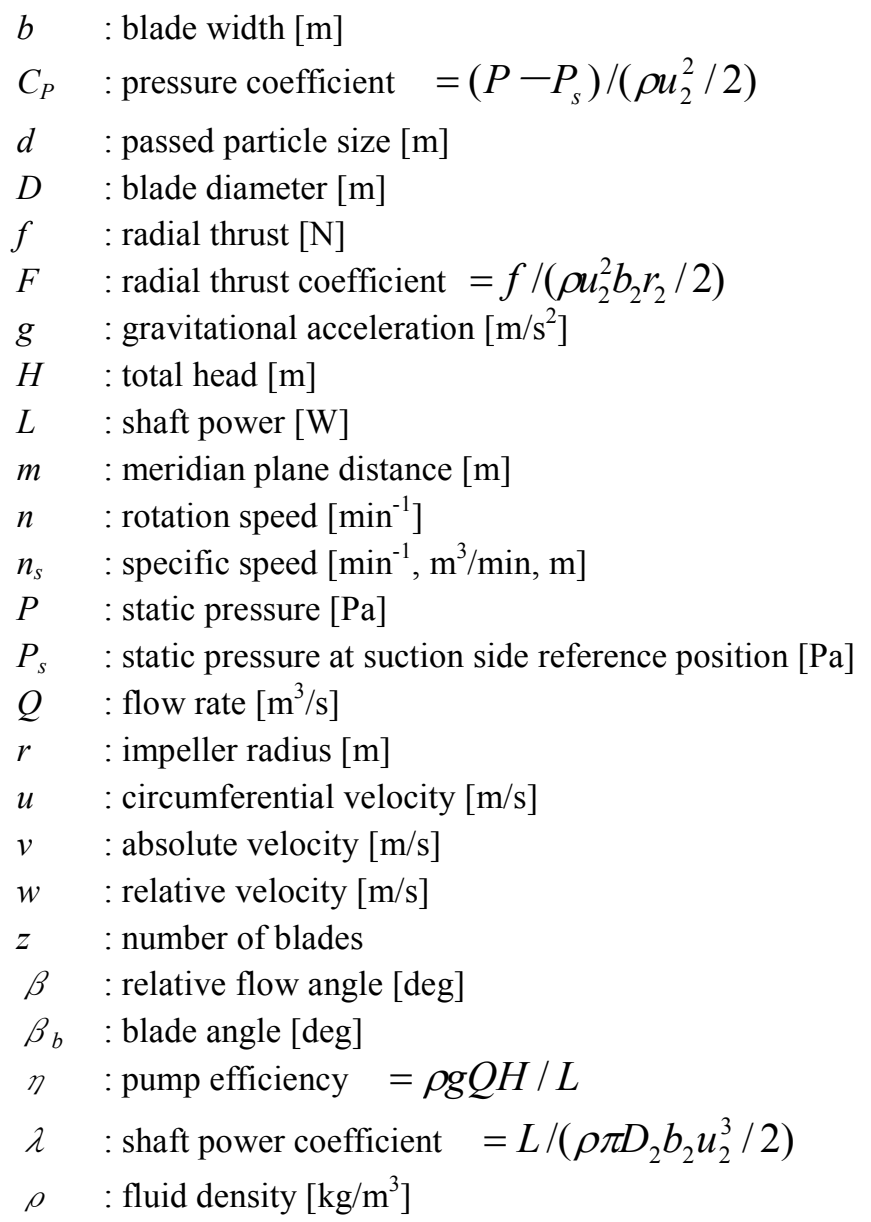




$$
\begin{array}{ll}
\phi & : \text { flow rate coefficient }=Q /\left(\pi D_{2} b_{2} u_{2}\right) \\
\phi & : \text { head coefficient }=H /\left(u_{2}^{2} / 2 g\right)
\end{array}
$$

Subscripts

th : theoretical value

$0,1,2$ : suction port; blade inlet; blade outlet

$m, u \quad$ : meridian direction component; circumferential component

$x, y \quad$ : x-direction component; y-direction component

\section{Method of Designing an Impeller}

Fig. 1 shows the design flow chart proposed by this study for the single-blade centrifugal impeller for which the passed particle size is specified. The feature of this design method is to use the relative velocity ratio $w_{2} / w_{1}$ that takes into account the slip at the blade outlet and suction velocity ratio $v_{m l} / v_{0}$ as design parameters. It is considered that, by using $w_{2} / w_{1}$ and $v_{m 1} / v_{0}$ expressing the macroscopic flow state from the suction inlet to the blade outlet of an impeller as design parameters, the method proposed by the authors is capable of establishing a more systematic impeller design method than the method in which major dimensions of an impeller from design diagrams are determined empirically. In the following, the detail of this design method will be presented.

When the flow rate $Q$, total head $H$, and rotation speed $n$ are given as design specifications, the specific speed $n_{s}$ can be obtained. On the basis of this $n_{s}$, the target pump efficiency $\eta$ is determined using past track records and similar data as bases. With the volumetric efficiency $\eta_{v}$ and mechanical efficiency $\eta_{m}$ estimated on the basis of past track records, the theoretical head $H_{t h}$ for a case of a finite number of blades is determined from the following equation:

$$
H_{t h}=\frac{\eta_{v} \eta_{m} H}{\eta}
$$

Used to determine the blade outer diameter $D_{2}$, the outlet circumferential velocity constant $K_{u 2}$ is the most important factor determining the performance of a pump. However, as Toyokura et al. ${ }^{(3)}$ has pointed out that, $K_{u 2}$ values of analogous pumps vary depending on the size of a pump since the total head $H$ changes depending on the pump efficiency. For this reason, this design method adopts the specific speed $n_{s t h}$ under the condition of a finite number of blades using $H_{t h}$ instead of $H$, as Toyokura et al. did, determining $n_{\text {sth }}$ from the following equation:

$$
n_{s t h}=n \frac{(60 Q)^{1 / 2}}{H_{t h}^{3 / 4}}
$$

Storing $K_{u 2}$ values for $n_{s t h}$ in the form of design diagrams in a database allows a $K_{u 2}$ value to be selected from a design diagram easily. Hence, the blade outer diameter $D_{2}$ can be determined from the following equation:

$$
D_{2}=\frac{60 K_{u 2} \sqrt{2 g H_{t h}}}{\pi n}
$$

When the passed particle size $d$ is specified as in the case of a sewage pump, the blade outlet width $b_{2}$ is under the relation of $b_{2} \geqq d$ and the value of $b_{2}$ is assumed within this range. When a specified passed particle size is large, giving consideration to avoid excessive velocity distortion in the width direction yields $b_{2}=d$.

It is necessary to select as small a number of blades $z$ so that a passed particle size $d$ may be secured in forming the shape of a flow channel between blades. When a large passed particle size is specified, a single-blade construction is adopted as a logical consequence. 


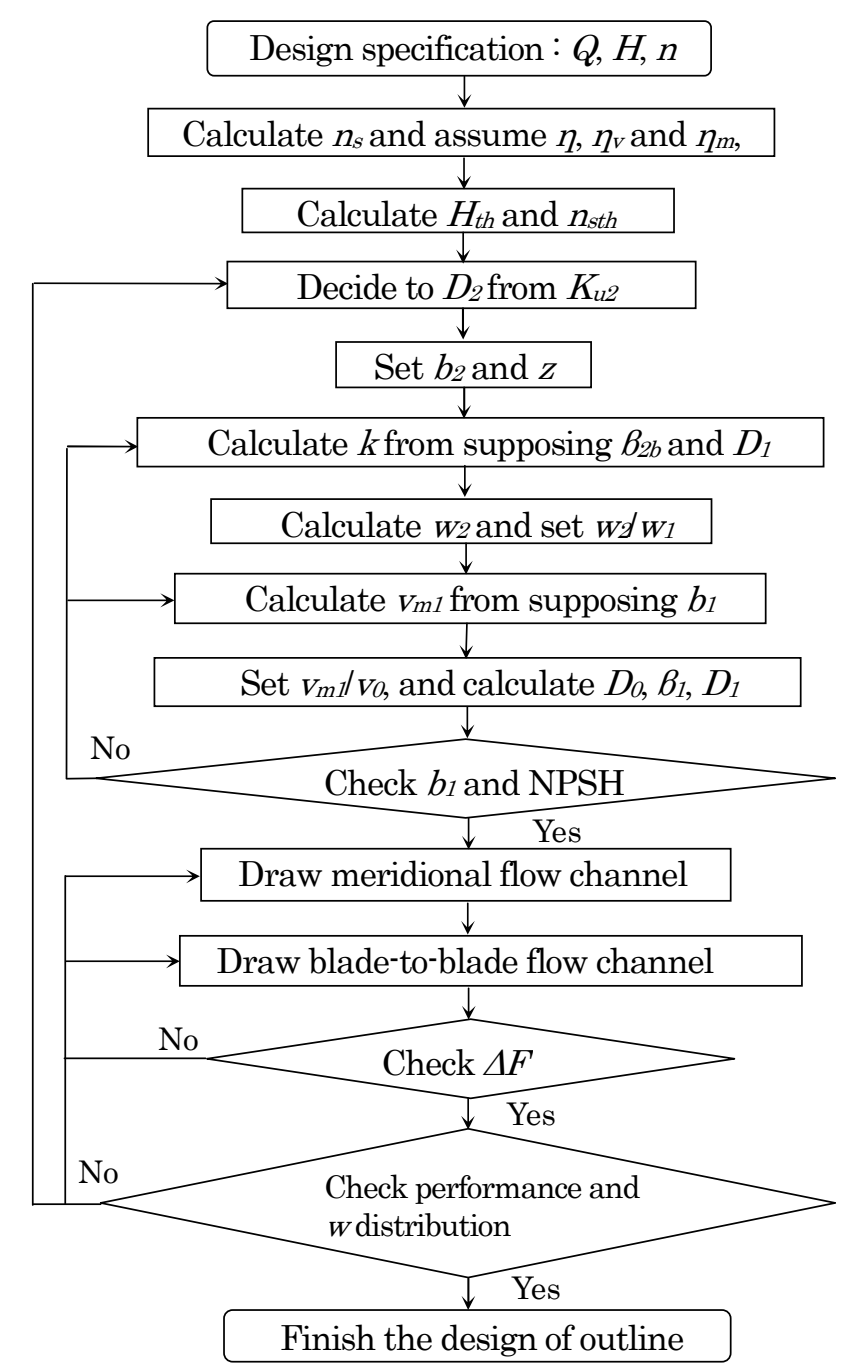

Fig. 1 Design flow

With $\sigma_{2}$ denoting the circumferential blade thickness at the blade outlet, the blade outlet meridian velocity $v_{m 2}$ taking the flow channel area reduction rate into consideration can be determined from the following equation:

$$
v_{m 2}=\frac{Q}{\eta_{v}\left(\pi D_{2}-Z \sigma_{2}\right) b_{2}}
$$

Next, the blade outlet angle $\beta_{2 b}$ is assumed. To consider the slip at the blade outlet, this study used Wiesner's formula ${ }^{(7)}$ to estimate the slip factor $k$. When the blade inner diameter $D_{l}$ is assumed to determine the slip coefficient $k$, the relative velocity at the blade outlet under the condition of a finite number of blades $w_{2}$ is given by the following equation:

$$
w_{2}=\sqrt{v_{m 2}^{2}+\left(k u_{2}+\frac{v_{m 2}}{\tan \beta_{2 b}}\right)^{2}}
$$

Flow in a pump impeller is usually "decelerating flow," but excessive deceleration causes boundary layers to be thickened and separation to occur. According to Stepanoff, the proper ratio of relative velocities at the inlet and outlet of a blade $w_{2} / w_{l}$ is 0.8 to $0.87^{(2)}$. But considering Stepanoff's design constants, Ohshima concluded that the outlet circumferential velocity $K_{u 2}$ and the outlet flow rate constant $K_{m 2}$ agree with the values calculated for a double suction type impeller with $w_{2} / w_{1}$ set at $0.65^{(8)}$. Mizuki et al. investigated the relation 
of the blade loading distribution to the flow state and performance of centrifugal compressors. They showed that flow is separated at the part with a large curvature on the shroud side with decelerating flow type impellers $\left(w_{2} / w_{1}=0.6\right)$ and that the effect of secondary flow is strong and loss increases with accelerating flow type impeller $\left(w_{2} / w_{l}=1.4\right)^{(9)(10)}$. Mishina investigated the relationship between the relative speed distribution and performance of centrifugal compressors. He showed that, regardless of the specific speed, flow shifts to the hub side and the impeller efficiency falls when the maximum decelerating velocity ratio decreases below the range of 0.625 to $0.645^{(11)(12)}$. This maximum decelerating velocity ratio may be controlled to a certain degree by changing the blade loading distribution, that is, the blade shape even under the same relative velocity ratio $w_{2} / w_{1}$. It is considered important in designing an impeller to provide a proper value of the relative velocity ratio $w_{2} / w_{1}$ as a macroscopic flow from the inlet to the outlet of a blade. In particular, it is considered that, due to a notable effect of slip in a single-blade centrifugal impeller, the relative velocity at the blade outlet $w_{2}$ is considerably larger than the relative velocity at the inlet $w_{1}$ and forms an accelerating flow. This requires the design to be performed with the relative velocity ratio considered in advance.

In response to this need, the relative velocity ratio $w_{2} / w_{1}$ is introduced as a design parameter. The value of $w_{2} / w_{1}$ is determined on the basis of past track records to determine the relative velocity at the blade inlet $w_{l}$.

For the blade inlet width $b_{l}$, the necessity of securing the passed particle size requires the relationship $b_{1} \geqq d$ to hold, with the $b_{1}$ value being selected from this range. When a specified passed particle size is large, giving consideration to avoid excessive velocity distortion in the width direction results in $b_{l}=d$.

With $\sigma_{1}$ denoting the circumferential blade thickness at the blade inlet, the blade inlet meridian velocity $v_{m l}$ taking the flow channel area reduction rate into consideration can be determined from the following equation:

$$
v_{m 1}=\frac{Q}{\eta_{v}\left(\pi D_{1}-Z \sigma_{1}\right) b_{1}}
$$

A single-blade centrifugal impeller used in a sewage pump must have a large blade inlet width to secure the necessary passed particle size, and as a result, the flow channel cross section of the blade inlet becomes too large in comparison with the flow channel cross section of the suction inlet. It is estimated that, for this reason, the fluid coming in from the suction inlet loses its velocity sharply near the blade inlet when it changes its direction from the axial to the radial direction, and uneven flow is generated as a result. It is estimated that this excessive reduction in velocity near the blade inlet is the cause of poor pump efficiency observed with conventional single-blade centrifugal impellers, and for this reason, it becomes necessary to consider the suction velocity ratio in designing impellers also.

In response to this need, the suction velocity ratio $v_{m l} / v_{0}$ is introduced as a design parameter. To determine the suction velocity $v_{0}$, the value of $v_{m l} / v_{0}$ is determined on the basis of past track records to avoid excessive reduction in velocity. Hence, the suction diameter $D_{0}$ can be determined from the following equation:

$$
D_{0}=\sqrt{\frac{4 Q}{\eta_{v} \pi v_{0}}}
$$

The blade inlet angle $\beta_{l b}$ is obtained from the following equation:

$$
\beta_{1 b}=\sin ^{-1}\left(\frac{v_{m 1}}{w_{1}}\right)
$$

Assuming that prewhirl is not present, the blade inner diameter $D_{l}$ can be obtained from the following equation: 


$$
D_{1}=\frac{60 v_{m 1}}{\pi n \tan \beta_{1 b}}
$$

The blade inlet width $b_{1}$ can therefore be obtained from the following equation:

$$
b_{1}=\frac{Q}{\eta_{v}\left(\pi D_{1}-Z \sigma_{1}\right) v_{m 1}}
$$

With $\beta_{2 b}, D_{l}$, and $b_{l}$ being modified, calculation is repeated until the $b_{l}$ value obtained from equation (10) converges on the assumed $b_{l}$.

The above calculations determine the major pieces of data for the impeller $D_{0}, D_{1}, D_{2}$, $b_{1}, b_{2}, \beta_{1 b}$, and $\beta_{2 b}$.

Using the pieces of data determined for the inlet, the required net positive suction head $H_{s v}$ is determined from the following equation to check for the possibility of cavitation:

$$
H_{s v}=\lambda_{1} \frac{w_{1}^{2}}{2 g}+\lambda_{2} \frac{v_{1}^{2}}{2 g}
$$

In the above equation, $\lambda_{1}$ and $\lambda_{2}$ denote the experimental coefficients of the respective velocity heads.

Next, a suitable meridian flow channel compatible with the specific speed $n_{\text {sth }}$ for a finite number of blades is created on the basis of the determined pieces of impeller data. This meridian flow channel is divided into several flow channels where the flow rates are the same ${ }^{(2)}$. In the range of $n_{s} \leqq 500$, using a total of three lines consisting of the central stream line and those on the hub plane and the shroud plane is considered sufficient for practical use.

Using the method of error triangles ${ }^{(2)}$, the blade angle distribution and the blade loading distribution over the dimensionless meridian plane distance $m / m_{2}$ are controlled on stream lines on the center, the hub, and the shroud plane to create flow channels between blades. However, it is necessary to adjust the blade winding angle in order to secure the passed particle size $d$ between blades.

Being geometrically asymmetrical, a single blade undergoes large fluctuating radial thrust. This radial thrust can be expressed as a vector sum of the averaged component (time-averaged value) based on the volute casing $F_{a v}$ and the fluctuating component based on the impeller itself $\Delta F^{(13)}$. At the best efficiency point flow rate, $F_{a v}$ is nearly zero, while $\Delta F$ depends on the circulation distribution of the impeller significantly ${ }^{(4)}$. Accordingly, it is necessary to identify blade loading distributions suitable to single blades with high $\eta$ and small $\Delta F$ by investigating the effect of blade loading distribution on performance characteristic and the fluctuating component of radial thrust $\Delta F$ for individual meridian flow channels.

Using the created center stream line for the blade shape as the typical line and the blade loading distribution for the blade winding angle, the fluctuating component of radial thrust $\Delta F$ is checked by means of the following method.

With the blade thickness being neglected in a one-dimensional incompressible flow, the fluid flows along the blade. Assuming that the impeller rotates in a field without restraints around, the following equation holds according to the law of conservation of angular momentum:

$$
\left[\left(r \rho Q v_{u}+\frac{\partial\left(r \rho Q v_{u}\right)}{\partial m} d r\right)-r \rho Q v_{u}\right]=z r\left(P_{+}-P_{-}\right) d r \cdot b
$$

Accordingly, the difference in pressure between the face and the back of a blade $P_{+}-P_{-}$ is given by the following equation:

$$
P_{+}-P_{-}=\rho \frac{Q}{z r b} \cdot \frac{\partial\left(r v_{u}\right)}{\partial m}
$$


In addition, the equation of continuity allows the following equation to hold:

$Q=2 \pi r b v_{m}$

The circulation distribution of an impeller $r v_{u}$ is given by the following equation:

$r v_{u}=r^{2} \omega-\frac{Q}{2 \pi b \cdot \tan \beta}$

Partially differentiating equation (15) with respect to $m$ gives the following equation:

$\frac{\partial\left(r v_{u}\right)}{\partial m}=2 r \omega \cdot \frac{\partial r}{\partial m}+\frac{Q}{2 \pi b \sin ^{2} \beta} \cdot \frac{\partial \beta}{\partial m}$

On the other hand, the radial thrust with a finite number of blades acting on them $F_{t h}$, and its $x$-direction component $F_{x t h}$ and $y$-direction component $F_{y t h}$ are expressed in the following equations, respectively:

$$
\begin{aligned}
& F_{t h}=\sqrt{\left|F_{x t h}\right|^{2}+\left|F_{y t h}\right|^{2}} \\
& F_{x t h}=-\int_{0}^{\theta_{a}}\left(P_{+}-P_{-}\right) \frac{r d \theta_{a}^{\prime}}{\cos \beta_{b}} b \cdot \cos \left(\beta_{b}+\theta_{a}^{\prime}\right) \\
& F_{y t h}=-\int_{0}^{\theta_{a}}\left(P_{+}-P_{-}\right) \frac{r d \theta_{a}^{\prime}}{\cos \beta_{b}} b \cdot \sin \left(\beta_{b}+\theta_{a}^{\prime}\right)
\end{aligned}
$$

Where $\theta_{a}$ denotes the winding angle from the blade inlet end to the outlet end and $\theta_{a}$, the winding angle from the blade outlet end to the inlet end, respectively.

On the basis of the above calculations, the distribution of the slip factor $k$ from the inlet to the outlet of the blade is used to determine the distribution of the relative flow angle $\beta$ in equation (16), and $F_{x t h}$ and $F_{y t h}$ are calculated from equations (18) and (19).

And to determine the radial thrust with an infinite number of blades for the case in which slip is not considered $F_{\text {th }}, \quad \beta$ in equation (16) is changed to $\beta_{b}$ to determine $\partial\left(r v_{u \infty}\right) / \partial m$, and $F_{x t h \infty}$ and $F_{y t h \infty}$ are calculated from equations (18) and (19).

Next, the relative velocity distributions of stream lines on the center, the hub, and the shroud plane are calculated using the difference in pressure between the face and the back of the blade $P_{+}-P_{-}$to check the quality of the relative velocity distributions.

Lastly, the pump performance is checked using one-dimensional performance prediction and similar means to conclude the schematic design.

\section{Experimental Apparatus and Method}

\subsection{Test Impeller}

Fig. 2 shows schematically the single-blade centrifugal impeller with the passed particle size of $56 \mathrm{~mm}$ designed and manufactured on the basis of the design method proposed by this study, with its pieces of design data shown in Table 1 . The $K_{u 2}, w_{2} / w_{1}$, and $v_{m 1} / v_{0}$ values in Table 1 were selected on the basis of the authors' experience. The target of the pump efficiency was set at $\eta=0.65$, with $\eta_{v}$ and $\eta_{m}$ estimated at 0.95 and 0.85 , respectively.

The distributions of the blade angle $\beta_{b}$ and the blade loading $\partial\left(r v_{u \infty}\right) / \partial m$ over the dimensionless meridian plane distance $m / m_{2}$ are shown in Figs. 3 and 4 . Regarding the three stream lines on the center, the hub, and the shroud plane, the distribution of $\beta_{b}$ was controlled as shown in Fig.3 so that the distribution of $\partial\left(r v_{u \infty}\right) / \partial m$ may change smoothly from the blade inlet to the outlet as shown in Fig.4.

\subsection{Experimental Apparatus and Method}

Fig.5 shows a diagrammatic sketch of the experimental apparatus. With the experiment carried out with the rotation speed set at $n=1740 \mathrm{~min}^{-1}$, the flow rate $Q$ was varied with a 
gate valve mounted to the discharge side and measured with an electromagnetic flow meter. The static pressure upstream and downstream the pump was measured with a strain gage type small pressure transducer to determine the total head $H$. The rotation speed $n$ was measured with an electromagnetic pickup and the driving torque $T$ with a torsion-bar-type torque detector to determine the shaft power $L$.

Fig. 6 shows the definition of the coordinate system and the measuring points of pressure fluctuation. The direction horizontal to the volute casing is defined as the $\mathrm{X}$-axis and the direction perpendicular to the volute casing as the Y-axis. With the impeller phase angle $\theta_{0}$ taken anticlockwise from the $\mathrm{X}$-axis, the point at which the blade outlet end passes the $\mathrm{X}$-axis (the position shown in Fig.6) is defined as $\theta_{0}=0^{\circ}$.

A total of eight points to measure pressure fluctuation are located at a distance of 10 $\mathrm{mm}$ outward from the blade outlet end, as shown in Fig.6, at intervals of $45^{\circ}$ in the anticlockwise direction with $\theta=0^{\circ}$ set on the X-axis. Pressure fluctuation was measured in synchronism with the rotation of the impeller by generating a trigger signal at the moment the blade outlet end is at $\theta_{0}=0^{\circ}$.

Radial thrust was measured with X-Y load cell incorporated into the bearing rim and in synchronism with the rotation of the impeller ${ }^{(4)}$. A strain gage connected with the four active gage method is glued to the X-Y load cell. To calibrate X-Y load cell, the dynamic calibration method $^{(14)}$ was adopted that verifies the relationship between the output voltage and force under the action of centrifugal force of a known magnitude. The influence of heat

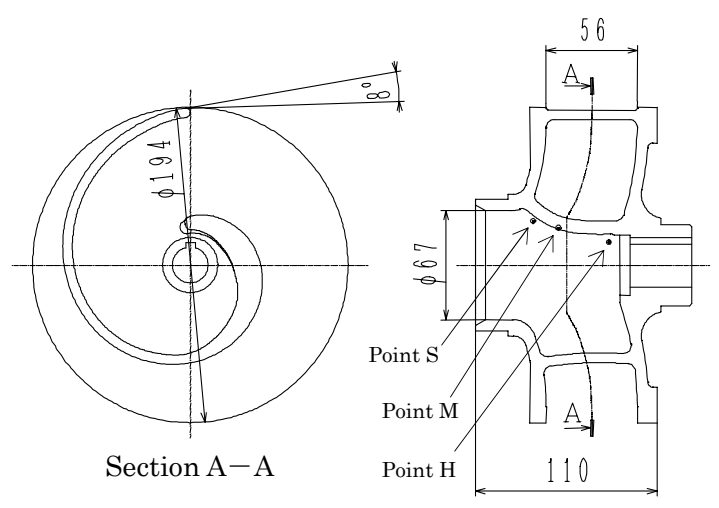

Fig. 2 Test impeller
Table 1 Specification of the impeller

\begin{tabular}{|c|c|}
\hline$Q$ & $0.7 \mathrm{~m}^{3} / \mathrm{min}$ \\
\hline$H$ & $11.5 \mathrm{~m}$ \\
\hline$n$ & $1728 \mathrm{~min}^{-1}$ \\
\hline \hline$K_{u 2}$ & 1.05 \\
\hline$W_{2} / W_{1}$ & 1.7 \\
\hline$V_{m 1} / V_{O}$ & 0.35 \\
\hline \hline$Z$ & 1 \\
\hline$D_{0}$ & $67 \mathrm{~mm}$ \\
\hline$D_{1}$ & $58 \mathrm{~mm}$ \\
\hline$D_{2}$ & $194 \mathrm{~mm}$ \\
\hline$b_{1}$ & $56 \mathrm{~mm}$ \\
\hline$b_{2}$ & $56 \mathrm{~mm}$ \\
\hline$\beta_{1 b}$ & 13 \\
\hline$\beta_{2 b}$ & 8 \\
\hline
\end{tabular}

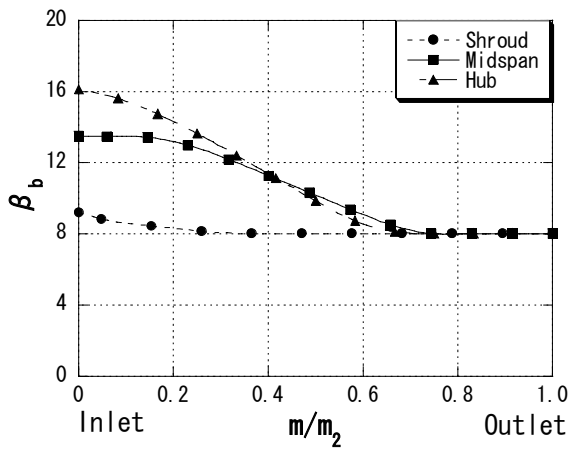

Fig. $3 \beta_{b}$ distributions

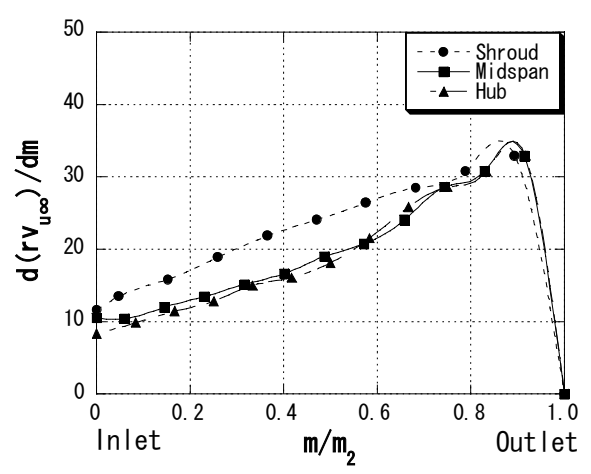

Fig. $4 \quad \partial\left(r v_{u \infty}\right) / \partial m$ distributions 


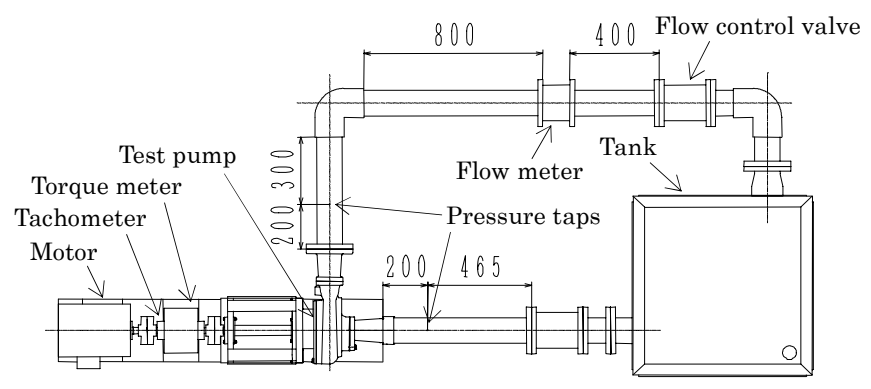

Fig. 5 Experimental apparatus

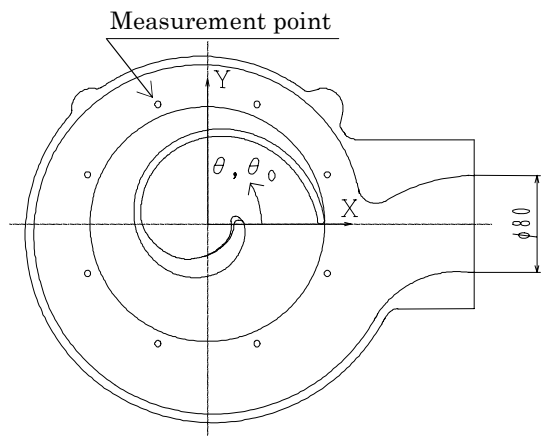

Fig. 6 Measurement points

on the bearing section was removed by means of a measuring method in which the pump is momentarily stopped under thermally stable condition to adjust the zero point and is immediately brought back to operation under the same condition ${ }^{(15)}$.

\section{Method and conditions of analysis}

To carry out CFD analysis, the FLUENT6.3 general-purpose analysis code was used to carry out three-dimensional unsteady flow analysis taking into consideration the mutual interaction between the impeller and the volute casing ${ }^{(5)(6)}$. In this analysis, the standard wall function was used to handle regions near wall surfaces and the standard $k-\varepsilon$ model was adopted as the turbulent flow model.

The computational domain consists of the suction pipe, the impeller, the volute casing, and the discharge pipe, with the gap between the impeller and the volute casing being not modeled. The total number of grids in the computational domain is about 900,000.

To give the boundary conditions, the inlet of the suction pipe is set to be at the inlet of the computational domain and the outlet of the discharge pipe at the outlet of the domain, with the mass flow rate in the absolute coordinate system given as the inlet boundary condition and a static pressure of $0 \mathrm{~Pa}$ as the outlet boundary condition. Since the fluid domain of the impeller is established in the rotational coordinate system, a rotational angular velocity in the opposite direction to that adopted in the experiment is given; the blade surface and the wall surfaces of the rear shroud and front shroud are defined as fixed walls in the relative coordinate system; and the suction pipe, the volute casing, and the discharge pipe are defined as fixed walls in the absolute coordinate system. To connect the outlet of the suction pipe to the suction inlet of the impeller and the outlet of the impeller to the inlet of the volute casing, the sliding mesh method ${ }^{(16)}$ was used. 


\section{Results of Experiment and Analysis and Discussion}

\subsection{Performance Characteristics}

Fig. 7 shows experimental and calculated performance curves of the single-blade centrifugal impeller designed by the proposed design method. The design point for the impeller is also shown in the figure. Taking note of the experimental values for the head coefficient $\phi$ allows one to see that the design specifications are satisfied by the original design. The pump efficiency $\eta$ attained $62 \%$, though falling a little short of the target of $65 \%$, being superior to $\eta$ values of conventional single-blade impeller ${ }^{(15)}$. However, the best efficiency is obtained near $\phi=0.030$, on the larger flow rate range than the design flow rate $\phi=0.019$. The possible reason for this is that, as shown in Fig. 8 , the matching point, which is the point of intersection of the theoretical head with a finite number of blades $H_{t h}$ and the volute characteristic equation ${ }^{(17)}$, is located on the larger flow rate than that of the impeller design point. The value of $H_{t h}$ is determined from the shaft power in the same way as the slip factor $k$ to be described later ${ }^{(4)}$.

As shown in Fig. 7, the experimental and calculated performance curves show a good agreement.

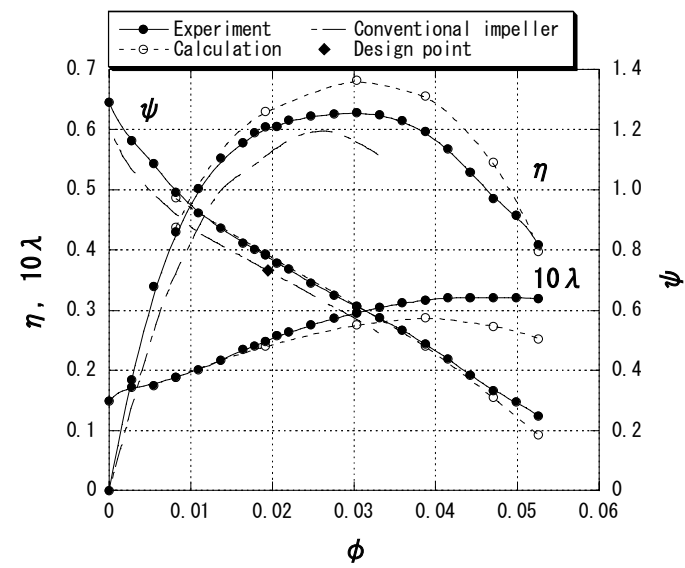

Fig. 7 Performance curves

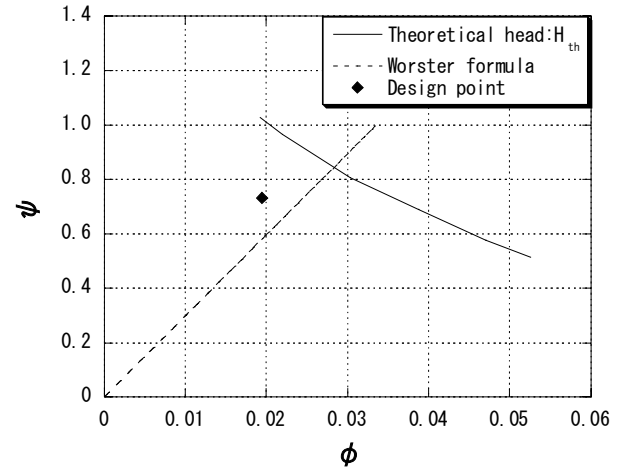

Fig. 8 Theoretical head and volute characteristics

\subsection{Design Parameters}

Table 2 compares the values of design parameters $w_{2} / w_{1}$ and $v_{m l} / v_{0}$ obtained by CFD analysis with the design values. The values obtained by CFD analysis shown in Table 2 were determined by using the mass flow averaged values for each flow channel cross section. To begin with, the comparison between the value of $v_{m l} / v_{0}$ obtained by CFD analysis and its design counterpart gives an error of $1.4 \%$, exhibiting a rough agreement. On the other hand, the value of $w_{2} / w_{1}$ obtained by CFD analysis is 2.51 , a considerably large value in comparison with the design value of 1.7. The discrepancies between the values of $w_{1} / u_{2}$ and $w_{2} / u_{2}$ obtained by CFD analysis and their design counterparts are $-28.9 \%$ and $5.0 \%$, respectively, showing that the errors in both of $w_{2} / w_{1}$ are mainly due to the difference in $w_{1} / u_{2}$.

Fig. 9 shows the results of CFD analysis on the instantaneous velocity distributions of $v_{m}$ and $v_{u}$ at the blade inlet under the design flow rate $\phi=0.019$. The measuring points were fixed on the absolute coordinate system, with three points provided on the $+x$-axis in Fig. 6 , the hub (Point H), the center (Point M), and the shroud (Point S) near the blade inlet. The relative positions for $\theta_{0}=0^{\circ}$ with reference to the blade are those shown in Fig.2. Fig. 9 shows that $v_{m}$ varies greatly at any measuring point due to the influence of the blade position and that a region in which $v_{m}$ shows negative values exists ahead of the blade inlet 
end. It is clearly shown that $v_{u}$ increases in this region and that a backflow region which rotates together with the impeller exists. As described above, this impeller adopts $v_{m 1} / v_{0}=0.35$ for the design value. Accordingly, it is considered that an excessive reduction in flow velocity even at the design flow rate results in the occurrence of a backflow region that rotates with the impeller.

The above consideration allows one to infer that the difference between the value of $w_{1} / u_{2}$ obtained by CFD analysis and their design counterpart is due to an increase in $v_{u l}$ as a result of the inlet flow being pulled into the backflow region that rotates with the impeller. In addition, it is estimated that an increase in $v_{u l}$ due to the finite blade thickness which is neglected the current design also caused the difference.

Table 2 Comparison of design parameter

\begin{tabular}{|c|c|c|c|}
\hline & CFD Calculation & Design value & Error (\%) \\
\hline \hline$W_{1} / u_{2}$ & 0.21707 & 0.30548 & -28.9 \\
\hline$W_{2} / u_{2}$ & 0.54529 & 0.51932 & 5.0 \\
\hline$W_{2} / W_{1}$ & 2.51 & 1.7 & 47.6 \\
\hline \hline$V_{0} / u_{2}$ & 0.19056 & 0.19585 & -2.7 \\
\hline$V_{m 1} / u_{2}$ & 0.06577 & 0.06854 & -4.0 \\
\hline$V_{m 1} / V_{0}$ & 0.345 & 0.35 & -1.4 \\
\hline
\end{tabular}

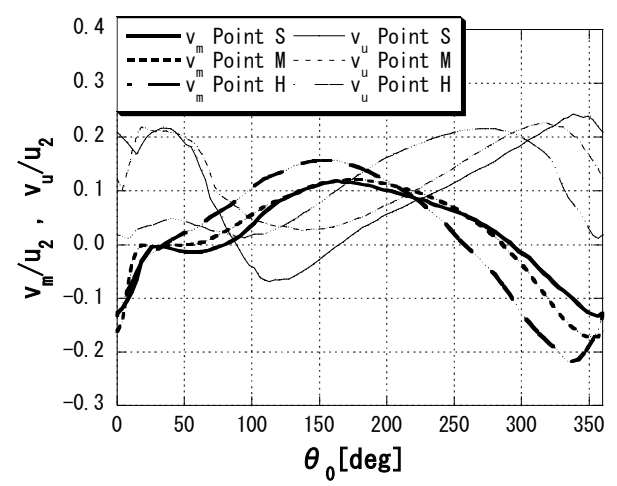

Fig. $9 v_{m}$ and $v_{u}$ distributions $(\phi=0.019)$

\subsection{Pressure Field}

Fig. 10 compares the experimental values of the circumferential pressure distribution averaged over time for each flow rate with the values obtained by CFD analysis. Both the experimental values and the values obtained by CFD analysis show that the pressure coefficients $C_{P}$ are roughly constant in the circumferential direction both at the design flow rate $\phi=0.019$ and at the best efficiency point flow rate $\phi=0.030$. In addition, both the experimental values and those obtained by CFD analysis show a similar trend indicating that $C_{P}$ at the volute winding end is larger on the smaller flow rate and $C_{P}$ at the volute winding end is smaller on the larger flow rate. Both agree well with each other at any flow rate.

Fig. 11 shows the instantaneous pressure distributions at measuring points $\theta=112.5^{\circ}$ and $247.5^{\circ}$ for the design flow rate $\phi=0.019$. The pressure coefficient $C_{P}$ decreases slowly as the blade pressure surface draws nearer to the measuring point, exhibiting the minimum value near the point at which the blade outlet end passes. This trend is captured by experiments and CFD analysis. A satisfactory agreement in the instantaneous pressure distribution between the experimental values and the CFD analysis values, as in the case of 


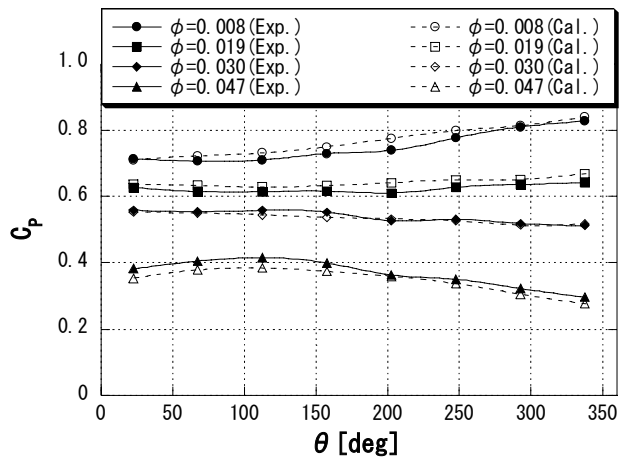

Fig. 10 Time averaged $C_{P}$ distributions

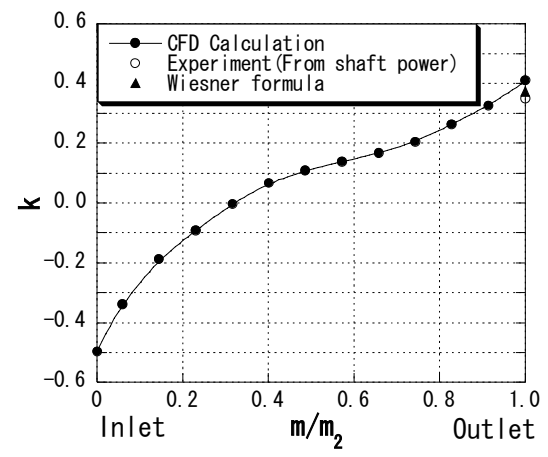

Fig. 12 Slip factor distribution

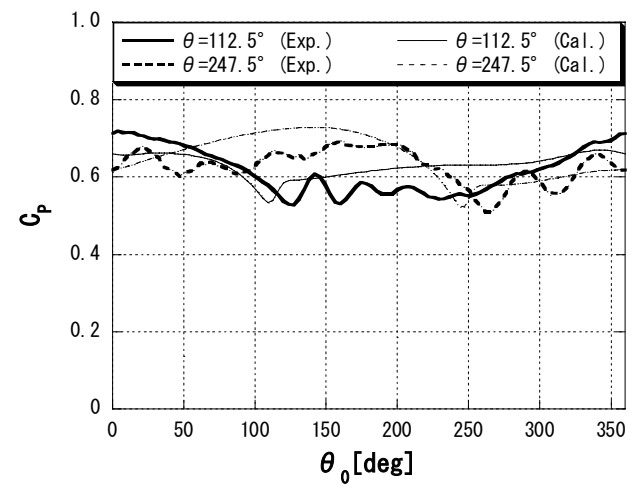

Fig. 11 Instantaneous $C_{P}$ distributions ( $\phi=0.019$ )

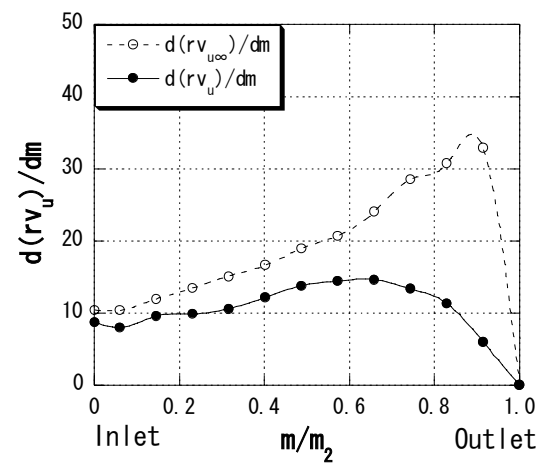

Fig. $13 \partial\left(r v_{u}\right) / \partial m$ distributions (Midspan)

the time-averaged pressure distribution, suggests that this analysis roughly reproduces the pressure field of this pump.

\subsection{Fluctuating Radial Thrust}

Fig. 12 shows the results of CFD analysis on the meridian plane distribution of the slip factor $k$ under the design flow rate $\phi=0.019$. The slip factor $k$ is obtained by defining it by means of the following equation:

$$
k=\left(v_{u \infty}-\overline{\overline{v_{u}}}\right) / u
$$

In the equation, $v_{u \infty}$ was calculated by using $\beta_{b}$ on the center stream line and also using the mass flow averaged value of $v_{u}$ in the cross section perpendicular to the radial direction as $\overline{\overline{v_{u}}}$. In addition, Fig. 12 shows, at $m / m_{2}=1.0$, the $k$ values calculated by means of the shaft-power-based method ${ }^{(4)}$ and Wiesner's formula ${ }^{(7)}$. Affected by the backflow region shown in Fig. 9, the $k$ value from CFD analysis exhibits negative values near the blade inlet. This $k$ value takes a positive value near $m / m_{2}=0.3$ and increases sharply starting roughly at $m / m_{2}=0.7$ toward the blade outlet. A comparison of $k$ obtained by CFD analysis, the value obtained from the shaft power, and the value obtained by Wiesner's formula shows a satisfactory agreement, indicating that Wiesner's formula can be applied to this impeller. When $z=1$, however, a large value of $\beta_{2 b}$ is accompanied by a considerably large value obtained by Wiesner's formula; therefore, careful application is required in the case of a single blade with a large $\beta_{2 b}$ value. In this way, this impeller is very susceptible to the effect of slip, which extends into the impeller interior. For this reason, the difference in the relative flow angle inside the impeller between a case with an infinite number of blades and one with a finite number of blades is very large, due to the difference of blade loading, namely the difference in pressure between the front and the back surfaces of the blade.

In connection with this, Fig. 13 shows a comparison of blade loading distribution between in the case with an infinite number of blades on the center stream line and in another case with a finite number of blades determined by using $k$ based on CFD analysis, 
over a range of $m / m_{2}$. It is clear from the figures that $\partial\left(r v_{u \infty}\right) / \partial m$ for an infinite number of blades increases on the blade outlet side, while $\partial\left(r v_{u}\right) / \partial m$ for a finite number of blades starts to decrease near $m / m_{2}=0.7$ in response to the behavior of $k$ shown in Fig. 12.

Fig. 14 shows the values of the radial thrust based on X-Y load cell at the design flow rate $\phi=0.019$, values obtained by CFD analysis, the predicted values $F_{\text {tho }}$ with an infinite number of blades, and the predicted values $F_{t h}$ with a finite number of blades. $F_{t h \infty}$ and $F_{t h}$ are estimated on the basis of equations (18) and (19). For each radial thrust, values at $\theta_{0}=0^{\circ}$, $90^{\circ}, 180^{\circ}$, and $270^{\circ}$ are expressed by symbols $\boldsymbol{O}, \boldsymbol{\Delta}, \boldsymbol{\square}$, and $\boldsymbol{\diamond}$, respectively. $F_{t h \infty}$ and $F_{t h}$ in Figs. 14 and 15, respectively, are drawn in a circle with the time-averaged value of experimental values at the center in the respective figures. A comparison between experimental values based on X-Y load cell and values obtained by CFD analysis reveals that the Lissajous figures obtained by CFD analysis exhibit roughly close to a perfect circle while the Lissajous figures of experimental values exhibit a slightly flat shape. The experimental values and the values obtained by CFD differ from each other slightly in the magnitude and direction of fluctuating components, but both show comparatively similar values. With the design flow rate $\phi=0.019$ that is smaller than the best efficiency point flow rate $\phi=0.030$, a similar trend is observed in which the time-averaged values of both shift toward the + Y-axis. Next, a comparison of experimental values with $F_{t h o}$ and with $F_{t h}$ shows that both experimental values and $F_{\text {tho }}$ exhibit roughly similar values both in the magnitude and direction of fluctuating components, while $F_{t h}$ exhibits considerably smaller values under the influence of slip shown in Fig.13. Since $F_{t h}$ is obtained by integrating the difference in pressure between the front and the back surfaces of a finite number of blades in the circumferential direction, $F_{t h}$ is considered to be closely related with the force created by the pressure around the impeller.

Accordingly, Fig. 15 shows a comparison of Lissajous figures of the fluid force between experimental values obtained from the pressure distribution around the impeller shown partly in Fig. 11, numerical values obtained by CFD analysis, and predicted values $F_{t h}$ with a finite number of blades. The forces of experimental values obtained from the pressure distribution and that of values obtained by CFD analysis are determined on the assumption that the wall static pressure on the shroud side is constant in the width direction. A comparison of these values shows that values obtained by CFD analysis are slightly larger but $F_{t h}$ are close to experimental values.

As discussed above, a rough estimation of the force arising from the pressure around the impeller can be obtained by means of the method of simplified prediction of fluctuating components of radial thrust incorporated in this design method. However, effects of other

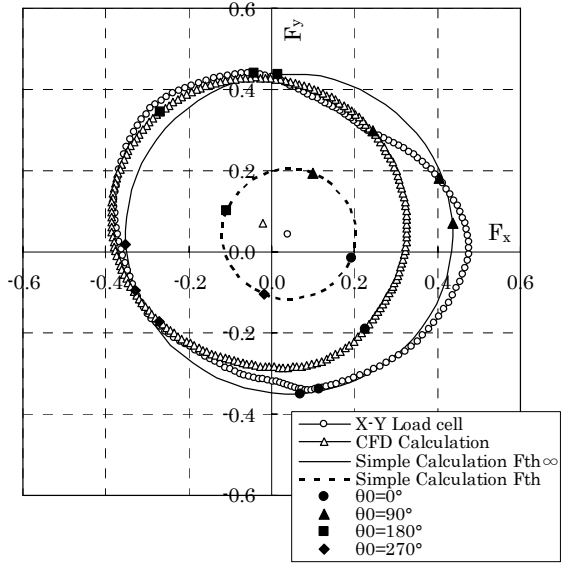

Fig. 14 Lissajous figures of force ( $\phi=0.019$ )

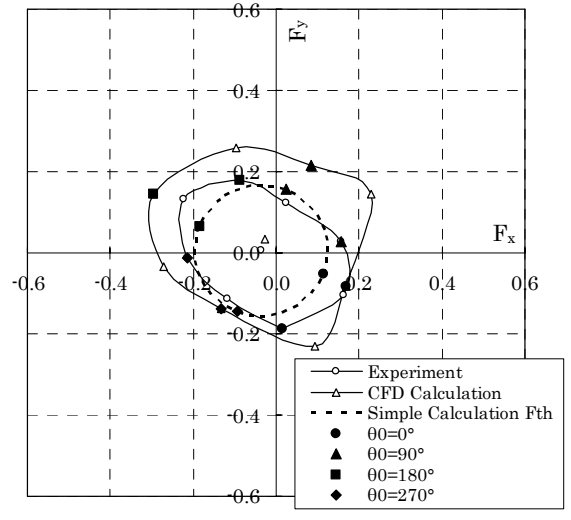

Fig. 15 Lissajous figures of force from pressure ( $\phi=0.019)$ 
forces in fluctuating components are large and the actual magnitude of fluctuating components is as large as $F_{t h \infty}$ with an infinite number of blades; this requires further improvement of simplified prediction.

\section{Conclusion}

A method of designing a single-blade centrifugal impeller for which the passed particle size is specified was proposed, and the experiment and CFD analysis based investigation of the performance and radial thrust of the single-blade centrifugal impeller designed by the proposed design method revealed the followings:

(1) The single-blade centrifugal impeller designed as described above satisfied the design point requirements, with the pump efficiency of $62 \%$ that exceeds the efficiency of conventional impeller.

(2) Regarding the suction velocity ratio $v_{m l} / v_{0}$, a design parameter introduced in this proposed design method, the design value and the value obtained by CFD analysis exhibited a good agreement. On the other hand, regarding the relative velocity ratio $w_{2} / w_{1}$ that is another design parameter, the relative velocities at the blade outlet agreed satisfactorily, while the relative velocities at the blade inlet exhibited a large difference under the influence of the backflow region near the blade inlet that rotates with the impeller.

(3) Predicted values of the fluctuating component of radial thrust with a finite number of blades, to be obtained by simplified prediction, were close to the force determined on the basis of pressure distribution. However, the force determined from the predicted value of the fluctuating component with a finite number of blades and from experimental pressure distribution is considerably smaller than experimental values based on X-Y load cell, suggesting that the influence other than the force due to the pressure around the impeller in the fluctuating component is large.

\section{References}

(1) Goto, A., Pump Design Using CFD Analysis and Inverse Design Method (in Japanese), Turbomachinery, Vol.28, No.11 (2000), p.641.

(2) Stepanoff, A. J., Centrifugal and Axial Flow Pumps, (1967), p.116, John Wiley \& Sons.

(3) Toyokura, T. and Takeda, H., New Design Charts for Turbo Pumps (in Japanese), Turbomachinery, Vol.23, No.8 (1995), p.41.

(4) Nishi, Y., Makita, K. and Fukutomi, J., A Study on a New Type of Sewage Pump (in Japanese), Transactions of the Japan Society of Mechanical Engineers, Series B, Vol.72, No.720 (2006), p. 1984.

(5) Nishi, Y., Matsuo, N. and Fukutomi, J., A Study on Internal Flow in a New Type of Sewage Pump (1st Report, Comparison between Experiment and CFD Calculation) (in Japanese), Transactions of the Japan Society of Mechanical Engineers, Series B, Vol.74, No.742 (2008), p. 1386.

(6) Nishi, Y., Matsuo, N. and Fukutomi, J., Loss Analysis of a New Type of Sewage Pump (in Japanese), Transactions of the Japan Society of Mechanical Engineers, Series B, Vol.74, No.744 (2008), p. 1785.

(7) Wiesner, F. J., A Review of Slip Factors for Centrifugal Impellers, Transactions of the American Society of Mechanical Engineers, Series A, Vol.89, No.4 (1967), p. 559.

(8) Oshima, M., Consideration about the Design Constants by Stepanoff for the Centrifugal Pump Impeller (in Japanese), Turbomachinery, Vol.29, No.4 (2001), p.221.

(9) Mizuki, S., Ariga, I. and Watanabe, I., Investigations Concerning the Optimum Blade Loading of Centrifugal Impeller (1st Report, In the Case of Static Pressure Distribution with in Impeller Channels) (in Japanese), Transactions of the Japan Society of Mechanical 
Engineers, Vol.41, No.342 (1975), p.502.

(10) Mizuki, S., Ariga, I. and Watanabe, I., Investigations Concerning the Optimum Blade Loading of Centrifugal Impeller (2nd Report, In the Case of Flow Patterns within Impeller Channels at the Design-Point) (in Japanese), Transactions of the Japan Society of Mechanical Engineers, Vol.41, No.342 (1975), p.515.

(11) Mishina, H., An Investigation of the Influence of the Relative Velocity Distribution on the Performance of Centrifugal Impellers (1st Report, Performance of High Specific Speed Impellers with Different Diffusion Ratios) (in Japanese), Transactions of the Japan Society of Mechanical Engineers, Series B, Vol.44, No.384 (1978), p.2689.

(12) Mishina, H., An Investigation of the Influence of Relative Velocity Distributions on the Performance of Centrifugal Impellers (2nd Report, Performance of Impellers with Different Specific Speeds and Blade Loading Profiles) (in Japanese), Transactions of the Japan Society of Mechanical Engineers, Series B, Vol.46, No.402 (1980), p.221.

(13) Aoki, M., Instantaneous Inter-Blade Pressure Distributions and Fluctuating Radial Thrust in a Single-Blade Centrifugal Pump (in Japanese), Transactions of the Japan Society of Mechanical Engineers, Series B, Vol.50, No.451 (1984), p.661.

(14) Tanaka, K., Arai, M., Ikeo, S. and Matsumoto, Y., Radial Thrust in Screw Centrifugal Pump Impeller (in Japanese), Turbomachinery, Vol.17, No.4 (1989), p.215.

(15) Ishida, I., The Consideration in the Radial Thrust Decrease of Centrifugal Pump (in Japanese), Turbomachinery, Vol.30, No.12 (2002), p.741.

(16) Fluent User's Manual (in Japanese), (2006), pp. 9-30.

(17) Worster, R. C., The Flow in Volutes and its Effect on Centrifugal Pump Performance, Proc. Instn. Mech. Engrs., Vol.177, No.31 (1963), p.843. 